\title{
Radiative Corrections to the Hadronic Cross-Section Measurement at DA $\Phi$ NE
}

\author{
V.A. Khoze $^{a)}$, M.I. Konchatnij ${ }^{b)}$, N.P. Merenkov ${ }^{b)}$, G. Pancheri ${ }^{c)}$, \\ L. Trentadue ${ }^{d)}$ and O.N. Shekhovzova ${ }^{b)}$
}

a) Department of Physics, University of Durham, Durham, DH1 3LE, England

b) National Science Centre "Kharkov Institute of Physics and Technology, 61108 Akademicheskaya 1, Kharkov, Ukrainia

c) INFN Laboratori Nazionali di Frascati, P.O. Box 13, I00044 Frascati, Italy

d) Dipartimento di Fisica, Universitá di Parma and INFN, Gruppo Collegato di Parma, 43100 Parma, Italy

\begin{abstract}
The hadronic invariant mass distribution for the process of electron-positron annihilation into a pair of charged pions accompanied by a photon radiated from the initial state has been studied for the region of DA $\Phi$ NE energies. The Born cross-section and the electromagnetic radiative corrections to it are calculated for realistic conditions of the KLOE detector. The dependence on the physical parameters which define the event selection is obtained.
\end{abstract}

\section{Introduction}

The idea to use radiative events in electron-proton and electron-positron interactions to expand the experimental possibilities for studies of different topics in high energy physics has become quite attractive in the last years. Different aspects of utilizing the radiative photons are now under intensive discussion.

Radiative events have been used already to measure the structure function $F_{2}\left(x, Q^{2}\right)$ at HERA [1]. The corresponding experimental setup takes advantage of a circle-shaped photon detector $(\mathrm{PD})$ in a very forward direction, as seen from the incoming electron beam. The PD measures the photon energy of all photons hitting it. The Born cross-section for such experimental conditions has been computed in [2], and further theoretical study has been performed to calculate the radiative corrections (RC) to the Born cross-section [3].

The possibility to undertake the $\Upsilon$-spectroscopy studies at the $\Upsilon(4 S)$ energy using the emission of a hard photon from the electron or positron has been considered in [4]. Estimates performed in this paper have demonstrated the feasibility of using the radiative photon events for the investigation of bottomonium spectroscopy at $B$-factories. 
Photon radiation from the initial $e^{+} e^{-}$-state in the events with missing energy has been successfully used at LEP for the measurement of the number of light neutrinos and for searches of new physics signals, see Ref. [5].

Recently, proposals to scan the hadronic cross-section $\sigma_{h}=\sigma\left(e^{+} e^{-} \rightarrow\right.$ hadrons $)$ at DAФNE energies through one such radiative process $[6,7]$ have been put forward. The strong motivation for such proposals lies in the fact that the measurement of $\sigma_{h}$, if performed below the one percent accuracy level, would allow an instructive test of the Standard Model via a precise determination of the anomalous magnetic moment $a_{\mu}=(g-2)_{\mu} / 2$ and the running electromagnetic coupling at the $Z$-peak $\alpha\left(M_{Z}^{2}\right)[7,8]$. On-going experiments in Brookhaven will soon reduce the experimental error on $a_{\mu}$ below the precision with which the electroweak contribution to this quantity is known, and could in principle make tests of new physics. Unfortunately, this cannot yet be envisaged, since the theoretical error on $a_{\mu}$ comes mainly from the uncertainty of the hadronic vacuum polarization contribution in the energy region below and around $1 \mathrm{GeV}$, where the hadronic contribution to the photon self energy cannot be calculated unambiguously within the framework of perturbative $Q C D$. Instead, this contribution is obtained via dispersion relations for the cross-section $\sigma\left(e^{+} e^{-} \rightarrow\right.$ hadrons $)$ [8,9]. A precise experimental determination of this quantity appears therefore the only means, at present, to reduce the theoretical error on $a_{\mu}$. There are two possible ways to measure unambiguously this cross-section in the energy region of interest, the direct scanning, as presently done at Novosibirsk [10], and the radiative return method. The radiative return method has a much smaller cross-section and, in order to have a statistical error in the necessary range, i.e. a fraction of a percent, it requires much more machine luminosity than the direct scanning, which is in principle the easiest to perform. Unfortunately, the reduction of the error through such direct measurement is not to be attained soon. Indeed, the precision attainable at VEPP2-M is limited by the machine luminosity, while DA $\Phi N E$, which has a much higher design luminosity, is planning to operate at the c.m. energy $\sqrt{s}=M_{\Phi}$ for the next few years. However, one can still make use of the planned DA $\Phi$ NE facility for this measurement in the near future, through the radiative return method, recently proposed as mentioned [6,7]. On the theoretical side, in order to reduce the systematic errors, it is necessary to perform radiative correction to at least the percent level to the process

$$
e^{-}\left(p_{1}\right)+e^{+}\left(p_{2}\right) \rightarrow \gamma(k)+\text { hadrons }(q) .
$$

When DA $\Phi$ NE operates at the $\Phi$-peak, the hadronic final state is dominated by the $\rho$-resonance decay products and the proposals to perform the experimental scanning of the $\pi^{+} \pi^{-} \gamma$ final state [6] to contribute to the reduction of the error on $a_{\mu}$ has made the detailed analysis of $\mathrm{RC}$ to process (1) a subject of theoretical efforts.

The Born cross-sections for the radiative process of electron-positron annihilation into a pair of charged fermions or scalar bosons were first calculated in [11]. This topics was subsequently considered in several papers, see, for example, Refs. [12,13].

In Ref.[14] the RC to total hadronic cross-section of process (1) with ISR were calculated analytically for the case when the PD measures the energies of all photons emitted in the narrow cone along the direction of the electron beam. These corrections include the first-order contribution with the next-to-leading accuracy and the high-order terms computing within the leading accuracy. At present such kind of PD is not the case for DA $\Phi$ NE. The KLOE detector allows to tag photons only outside a blind zone defined by two narrow cones along both, electron and positron beam directions. In addition, events with two hard photons tagged by the PD are rejected. Therefore, generally speaking the RC depend on "soft" (because of the photon energy selection in the PD) and "collinear" (because of the PD geometry) radiation 
parameters. Note that, as discussed in Section 3, the Born cross-section depends on collinear parameters only.

An analytical calculation of the first-order $\mathrm{RC}$ to the distribution over the tagged photon energy for the KLOE-type detector has been performed in [15], and an analysis of the $\pi^{+} \pi^{-} \gamma$ final state has been carried out in [16] using the Monte Carlo event generator for the evaluation of the $\mathrm{RC}$ given in [17].

The calculations of the RC performed in [15] do not take into account some specific (but essential) details of the event selection in the proposed experiment with the KLOE detector [6]. In this paper we calculate the distribution over the hadronic invariant mass in process (1) in the Born approximation and compute analytically the $\mathrm{RC}$ to this distribution accounting for the cuts discussed in Section 2.

\section{Event selection in KLOE}

The KLOE detector allows to measure independently the energy of the photon $\omega$ with the calorimeters (QCAL and EMCAL) and the invariant mass of the charged pions $q^{2}$ with the drift chamber (DC). The strategy of the experiment will be based on the measurements of the $q^{2}$ of the two pions with the DC which indirectly allows to reconstruct $\omega$. The much higher accuracy of the DC measurements as compared to the finite resolution of the electromagnetic calorimeter (EMCAL) is the basis for such a strategy. An attractive advantage of this approach is that, in principle, it does not require corrections of the measured distributions for the effects, caused by the experimental resolution of the calorimeter, the so-called deconvolution procedure, see Ref. [7].

Let us define the total 4-momentum of the initial electron and positron as

$$
p_{1}+p_{2}=\left(2 E, \vec{P}_{\Phi}\right)
$$

where $E$ is the beam energy, $\vec{P}_{\Phi}$ is the momentum of the $\Phi$ and $\left|\vec{P}_{\Phi}\right|=12.5 \mathrm{MeV}$ in the $X-$ direction [6]. Note that in the laboratory frame the Lorentz boost of the $\Phi$ is accounted for. In the interaction point the electron and positron exercise not exactly a head-on collision but there is a small beam crossing angle of order $\left|\vec{P}_{\Phi}\right| / 2 E$ relative to the $Z$ axis and

$$
p_{1}=\left(E, \frac{\left|\vec{P}_{\Phi}\right|}{2}, 0, P_{z}\right), \quad p_{2}=\left(E, \frac{\left|\vec{P}_{\Phi}\right|}{2}, 0,-P_{z}\right), \quad P_{z}=E\left(1-\frac{\left|\vec{P}_{\Phi}\right|^{2}}{8 E^{2}}\right) .
$$

Here we define the $X Z$ as the $\left(\vec{p}_{1}, \vec{p}_{2}\right)$ - plane, and $Z$ as the symmetry axis of the PD.

In spite of its smallness, the quantity $\left|\vec{P}_{\Phi}\right| / E$ should be taken into account in a high precision determination of the photon energy and in the calculation of the cross-section of process (1).

In the single photon emission events the photon energy $\omega$ can be reconstructed directly from $q^{2}=\left(p_{1}+p_{2}-k\right)^{2}$

$$
\omega=\frac{4 E^{2}-\left|\vec{P}_{\Phi}\right|^{2}-q^{2}}{2\left(2 E-\left|\vec{P}_{\Phi}\right| \sin \theta \cos \varphi\right)},
$$

where $\theta(\varphi)$ is polar (azimuthal) angle of a photon in the laboratory frame. We see that because of the difference between the laboratory and the centre-of-mass frames one can reconstruct the photon energy in (1) only if the exact angular orientation of the emitted photon is known. 
A systematic error could arise due to the events with the multiple photon emission. In order to reject these events as well as to decrease the background caused by the final-state radiation the following event selection cuts are imposed [6]

$$
\Omega-|\vec{K}| \leq \eta, \omega \geq \omega_{m}, \omega_{m}=50 \mathrm{MeV}, \quad \eta=10 \mathrm{MeV}
$$

where $\Omega(\vec{K})$ is the energy (3-momentum) of all emitted photons, assuming that only one hard photon with the energy $\omega$ is tagged by the PD. Here $\omega_{m}$ denotes threshold energy for this photon. These restrictions are based on the predictions of the Monte Carlo events generator described in Ref. [16].

The first inequality in (4) is the reduced form of the constraint

$$
M_{\Phi}-E_{+}-E_{-}-\left|\vec{P}_{\Phi}-\vec{p}_{+}-\vec{p}_{-}\right|<\eta,
$$

where $M_{\Phi}$ is the mass of $\Phi$-meson and $E_{+,-}\left(\vec{p}_{+,-}\right)$is the energy (3-momentum) of $\pi^{+}, \pi^{-}$for $\pi^{+} \pi^{-}+n \gamma$ events with $n \geq 1$.

Photon tagging by the QCAL calorimeter, which surrounds the blind zone and covers the angles from $\theta_{m}$ up to $20^{\circ}$ with respect to the electron beam direction as well as the symmetrical angles along the positron beam, can be done for the photon energy above the threshold $\omega_{\text {min }}^{q c}=$ $1 \mathrm{MeV}$ (here $\theta_{m}$ is the aperture of the blind zone ). The corresponding threshold for the EMCAL calorimeter, which covers the angles from $20^{\circ}$ up to $40^{\circ}$ with respect to both the electron and the positron beam directions, is $\omega_{\text {min }}^{e c}=5 \mathrm{MeV}$ ) [6]. As we noted above, the events with the two hard photons inside the PD are assumed to be rejected. Therefore, when calculating the RC, one has to take into account the possibility that a soft photon with the energy $\omega_{1}$ (additional to the tagged one) hits the detector, but is not registered. Thus, we can write the following constraints on the energy $\omega_{1}$ and the radiation angle $\theta_{1}$ of an additional soft photon inside the detector

$$
\begin{gathered}
\omega_{1}<\Delta_{1} E, \text { if } 160^{\circ}<\theta_{1}<\pi-\theta_{m} \text { and } \theta_{m}<\theta_{1}<20^{\circ}, \Delta_{1}=\frac{\omega_{\min }^{q c}}{E} \simeq 0.2 \cdot 10^{-2} ; \\
\omega_{1}<\Delta_{2} E \text {, if } 40^{\circ}>\theta_{1}>20^{\circ}, \text { and } 160^{\circ}>\theta_{1}>140^{\circ}, \Delta_{2}=\frac{\omega_{m i n}^{e c}}{E} \simeq 10^{-2}
\end{gathered}
$$

where $\theta_{m}$ is for about $10^{\circ}$ and $\theta_{1}$ is defined relative the $\mathrm{Z}$ axis.

In the following Section, we shall compute the distribution over the hadronic invariant mass in process (1) in the Born approximation.

\section{Born approximation}

To lowest order in $\alpha$, the differential cross-section for process (1) with respect to the tagged hard photon has been calculated in [11], and here we reproduce the expression for an arbitrary hadronic final state.

The general formula for the differential cross-section in the Born approximation can be written as

$$
d \sigma^{B}=\frac{2 \pi^{2} \alpha^{2}}{S}|M|^{2} \frac{\alpha}{4 \pi^{2}} \frac{d^{3} k}{\omega} d \Gamma,
$$

where $\alpha$ is the electromagnetic coupling, $S=2\left(p_{1} p_{2}\right)$ and

$$
d \Gamma=(2 \pi)^{4} \delta\left(q-\sum q_{f}\right) \prod \frac{d^{3} q_{f}}{2 \varepsilon_{f}(2 \pi)^{3}}
$$


is the phase space factor for the final hadrons, $q_{f}$ is the 4-momentum of an individual hadron. The squared matrix element on the right-hand side of Eq.(6) can be written in terms of the electronic and hadronic tensors $L_{\mu \nu}^{\gamma}$ and $H_{\mu \nu}$ as

$$
|M|^{2}=\frac{4}{q^{4}} L_{\mu \nu}^{\gamma} H_{\mu \nu}
$$

The subscript $\gamma$ in the electronic current tensor indicates that here we are dealing with ISR events in process $(1)$.

The differential cross section for radiative events can be obtained by integrating over all hadronic final states. This can be performed by using the well known relation

$$
\sum_{h} \int H_{\mu \nu}(q) d \Gamma=F_{h}\left(q^{2}\right) \widetilde{g}_{\mu \nu}, \quad \widetilde{g}_{\mu \nu}=g_{\mu \nu}-\frac{q_{\mu} q_{\nu}}{q^{2}}
$$

where the function $F_{h}\left(q^{2}\right)$ carries all the information about the non-radiative hadronic crosssection $\sigma_{h}\left(q^{2}\right)$. For the case of annihilation into a charged pion pair

$$
F_{h}\left(q^{2}\right)=\frac{q^{2}\left|F_{\pi}\left(q^{2}\right)\right|^{2}}{24 \pi}\left(1-\frac{4 \mu^{2}}{q^{2}}\right)^{3 / 2}
$$

where $\mu$ is the pion mass and $F_{\pi}\left(q^{2}\right)$ is the pion electromagnetic form factor.

The leptonic tensor can be presented as $[11,18]$

$$
L_{\mu \nu}^{\gamma}=\frac{\left(S+T_{1}\right)^{2}+\left(S+T_{2}\right)^{2}}{T_{1} T_{2}} \widetilde{g}_{\mu \nu}+\frac{4 q^{2}}{T_{1} T_{2}}\left(\tilde{p}_{1 \mu} \tilde{p}_{1 \nu}+\tilde{p}_{2 \mu} \tilde{p}_{2 \nu}\right), \quad \tilde{p}=p-\frac{p q}{q^{2}} q,
$$

where we introduced the following notations

$$
\begin{gathered}
T_{1}=-2 p_{1} k=-\omega\left(2 E-2 P_{z} \cos \theta-\left|\vec{P}_{\Phi}\right| \sin \theta \cos \varphi\right), \\
T_{2}=-2 p_{2} k=-\omega\left(2 E+2 P_{z} \cos \theta-\left|\vec{P}_{\Phi}\right| \sin \theta \cos \varphi\right), \\
S=2 p_{1} p_{2}=4 E^{2}-\left|\vec{P}_{\Phi}\right|^{2}, \quad q^{2}=S+T_{1}+T_{2} .
\end{gathered}
$$

In the expression for the leptonic tensor we have neglected terms of relative order $m^{2} /\left|T_{1}\right|$ and $m^{2} /\left|T_{2}\right|$ (here $m$ is the electron mass) because for the KLOE detector these cannot exceed $m^{2} /\left(E^{2} \theta_{m}^{2}\right) \simeq 10^{-4}$.

Taking into account that

$$
L_{\mu \nu}^{\gamma} \widetilde{g}_{\mu \nu}=2 \frac{\left(S+T_{1}\right)^{2}+\left(S+T_{2}\right)^{2}}{T_{1} T_{2}}
$$

we can present the Born cross-section as (see also Ref. [11])

$$
d \sigma^{B}=\sigma\left(q^{2}\right) \frac{\alpha}{2 \pi^{2}} \frac{\left(S+T_{1}\right)^{2}+\left(S+T_{2}\right)^{2}}{T_{1} T_{2}} \frac{d^{3} k}{S \omega}, \sigma\left(q^{2}\right)=\frac{\pi \alpha^{2}\left|F_{\pi}\left(q^{2}\right)\right|^{2}}{3 q^{2}}\left(1-\frac{4 \mu^{2}}{q^{2}}\right)^{\frac{3}{2}} .
$$

Let us multiply the right-hand side of Eq.(11) by

$$
d q^{2} \delta\left(4 E(E-\omega)-\left|\vec{P}_{\Phi}\right|^{2}-q^{2}+2 \omega\left|\vec{P}_{\Phi}\right| \sin \theta \cos \varphi\right)
$$


and use the $\delta$-function to perform the integration over $d \omega$. Imposing the threshold restriction (4) for the events with the emission of a single photon

$$
\omega \geq \omega_{m}
$$

we arrive at

$$
\begin{gathered}
\frac{d \sigma^{B}}{d q^{2}}=\frac{\alpha}{2 \pi^{2}} \sigma\left(q^{2}\right) \frac{\left(S-q^{2}\right) d \cos \theta d \varphi}{4 S\left(2 E-\left|\vec{P}_{\Phi}\right| \sin \theta \cos \varphi\right)^{2}} \frac{\left(S+T_{1}\right)^{2}+\left(S+T_{2}\right)^{2}}{T_{1} T_{2}} \\
\Theta\left(\frac{S-q^{2}}{2\left(2 E-\left|\vec{P}_{\Phi}\right| \sin \theta \cos \varphi\right)}-\omega_{m}\right) .
\end{gathered}
$$

In principle one can perform the angular integration on the right-hand side of Eq.(12) numerically. The analytical integration is complicated because of the $\Theta$-function. This results in nontrivial limits for the angular integration. To derive them let us first examine the quantity

$$
D=\frac{4 E\left(E-\omega_{m}\right)-q^{2}-\left|\vec{P}_{\Phi}\right|^{2}}{2 \omega_{m}\left|\vec{P}_{\Phi}\right|} .
$$

If $D>1$ then the emission of a photon is allowed in all available angular phase space. For

$$
1>D>\sin \theta_{m}
$$

two options appear, namely

$$
2 \pi>\varphi>0, \quad \arcsin D>\theta>\theta_{m}, \pi-\theta_{m}>\theta>\pi-\arcsin D
$$

and

$$
\arccos \frac{-D}{\sin \theta}>\varphi>0,2 \pi>\varphi>2 \pi-\arccos \frac{-D}{\sin \theta}, \pi-\arcsin D>\theta>\arcsin D .
$$

When $\sin \theta_{m}>D>-\sin \theta_{m}$ or $-\sin \theta_{m}>D>-1$ the limits for the azimuthal integration are the same as in Eq.(14) but the polar angles are different

$$
\sin \theta_{m}>D>-\sin \theta_{m}, \pi-\theta_{m}>\theta>\theta_{m},
$$

whereas at

$$
-\sin \theta_{m}>D>-1, \pi-\arcsin (-D)>\theta>\arcsin (-D) .
$$

Single photon emission with energy $\omega>\omega_{m}$ is not allowed if

$$
D<-1
$$

Using now the angular constraints given by Eqs.(13)-(16) one can perform the angular integration in Eq.(12) analytically (at least over the azimuthal angle). The result can be presented in the following form

$$
\frac{d \sigma^{B}}{d q^{2}}=\frac{d \sigma^{B}(D>1)}{d q^{2}}+\frac{d \sigma_{a}^{B}\left(1>D>s_{m}\right)}{d q^{2}}+\frac{d \sigma_{r}^{B}}{d q^{2}} .
$$


The quantity $d \sigma^{B}(D>1) / d q^{2}$ corresponds to events with $D>1$ when all radiation angles for the tagged photon are allowed. It reads

$$
\begin{gathered}
\frac{d \sigma^{B}(D>1)}{d q^{2}}=\frac{\alpha}{2 \pi} \frac{\sigma\left(q^{2}\right)}{2 E^{2}}\left\{\left(\frac{S}{S-q^{2}}-1+\frac{S-q^{2}}{2 S}\right)\left[2 \ln \frac{1+c_{m}}{1-c_{m}}+\frac{\left|\vec{P}_{\Phi}\right|^{2}}{2 E^{2}}\left(\ln \frac{1+c_{m}}{1-c_{m}}+\frac{c_{m}}{s_{m}^{2}}\right)\right]\right. \\
\left.-\frac{S-q^{2}}{S} c_{m}\left[1+\frac{\left|\vec{P}_{\Phi}\right|^{2}}{8 E^{2}}\left(3-c_{m}^{2}\right)\right]\right\}
\end{gathered}
$$

where $c_{m}=\cos \theta_{m}, s_{m}=\sin \theta_{m}$. The quantity $d \sigma_{a}^{B}\left(1>D>s_{m}\right) / d q^{2}$ describes events with $1>D>s_{m}$ for which full coverage in the azimuthal angle is allowed. It has a structure which is very close to the right-hand side of Eq.(18) and reads

$$
\begin{gathered}
\frac{d \sigma_{a}^{B}\left(1>D>s_{m}\right)}{d q^{2}}=\frac{\alpha}{2 \pi} \frac{\sigma\left(q^{2}\right)}{2 E^{2}}\left\{( \frac { S } { S - q ^ { 2 } } - 1 + \frac { S - q ^ { 2 } } { 2 S } ) \left[2 \ln \frac{\left(1+c_{m}\right)\left(1-c_{d}\right)}{\left(1-c_{m}\right)\left(1+c_{d}\right)}+\right.\right. \\
\left.\left.\frac{\left|\vec{P}_{\Phi}\right|^{2}}{2 E^{2}}\left(\ln \frac{\left(1+c_{m}\right)\left(1-c_{d}\right)}{\left(1-c_{m}\right)\left(1+c_{d}\right)}+\frac{c_{m}}{s_{m}^{2}}-\frac{c_{d}}{s_{d}^{2}}\right)\right]-\frac{S-q^{2}}{S}\left(c_{m}-c_{d}\right)\left[1+\frac{\left|\vec{P}_{\Phi}\right|^{2}}{8 E^{2}}\left(3-c_{m}^{2}-c_{d}^{2}-c_{m} c_{d}\right)\right]\right\},
\end{gathered}
$$

where $s_{d}=D, c_{d}=\sqrt{1-D^{2}}$.

The contribution of the remaining regions (see Eqs.(14), (15) and (16)) is described by the quantity $d \sigma_{r}^{B} / d q^{2}$. We integrate it only over the azimuthal angle and arrive at

$$
\begin{aligned}
\frac{d \sigma_{r}^{B}}{d q^{2}}= & \frac{\alpha}{\pi} \sigma\left(q^{2}\right) d \cos \theta\left[\left(\frac{S}{S-q^{2}}-1+\frac{S-q^{2}}{2 S}\right) \frac{2}{\pi P_{z} \cos \theta}\left(\Phi_{-}-\Phi_{+}\right)-\right. \\
& \left.\frac{S-q^{2}}{\pi S\left(4 E^{2}-\left|\vec{P}_{\Phi}\right|^{2} \sin ^{2} \theta\right)}\left(\frac{\left|\vec{P}_{\Phi}\right| \sqrt{\sin ^{2} \theta-s_{d}^{2}}}{2 E-\left|\vec{P}_{\Phi}\right| s_{d}}+4 E \Phi\right)\right] \Theta_{r},
\end{aligned}
$$

where

$$
\Phi_{ \pm}=\frac{1}{\sqrt{\left(2 E \pm 2 P_{z} \cos \theta\right)^{2}-\left|\vec{P}_{\Phi}\right|^{2} \sin ^{2} \theta}} \arctan \sqrt{\frac{\left(2 E \pm 2 P_{z} \cos \theta+\left|\vec{P}_{\Phi}\right| \sin \theta\right)\left(\sin \theta+s_{d}\right)}{\left(2 E \pm 2 P_{z} \cos \theta-\left|\vec{P}_{\Phi}\right| \sin \theta\right)\left(\sin \theta-s_{d}\right)}} .
$$

Note that on the right-hand side of Eq.(20) $\sin \theta$ always exceeds $s_{d}$. The quantity $\Phi$ can be obtained from $\Phi_{+}\left(\right.$or $\left.\Phi_{-}\right)$by

$$
\Phi=\Phi_{ \pm}\left(P_{z}=0\right) .
$$

The function $\Theta_{r}$ on the right-hand side of Eq.(20) is introduced to define the upper limits of the variable $\cos \theta$ at different values of $D$. It can be written explicitly as

$$
\begin{gathered}
\Theta_{r}=\theta\left(c_{d}-\cos \theta\right)\left[\theta(1-D) \theta\left(D-s_{m}\right)+\theta\left(-s_{m}-D\right) \theta(D+1)\right]+ \\
\theta\left(c_{m}-\cos \theta\right) \theta\left(s_{m}-D\right) \theta\left(s_{m}+D\right)
\end{gathered}
$$

provided that the minimal value of $\cos \theta$ equals to zero. One can verify that in the limit $\left|\vec{P}_{\Phi}\right|=0$ when only the region $D>1$ contributes (all emission angles are allowed) the right-hand side of Eq.(17) coincides with the well known expression, see, for example, Refs. $[15,16]$

$$
\frac{d \sigma^{B}}{d x}=\frac{\alpha}{2 \pi} \sigma\left(q^{2}\right) 2\left[\frac{1+(1-x)^{2}}{x} \ln \frac{1+c_{m}}{1-c_{m}}-x c_{m}\right]
$$

where in this limit $x=\omega / E=\left(4 E^{2}-q^{2}\right) /\left(4 E^{2}\right)$.

Note that an account for the $\left|\vec{P}_{\Phi}\right| / E$ effects is mainly essential for the reconstruction of the tagged photon energy (see Eq.(3)) if one wishes to guarantee the one percent accuracy level. 


\section{Radiative corrections}

The proposed high accuracy measurement of the pion contribution to the hadronic crosssection at $D A \Phi N E[6]$ by using radiative events in process (1), requires an adequately high precision of the theoretical predictions. These have to take into account at least the first-order QED radiative corrections. The first-order $\mathrm{RC}$ to $d \sigma^{B} / d q^{2}$ include the real and virtual soft photon contributions in the overall phase space as well as the hard real contribution from the region where the PD does not tag a photon. Since the effect caused by the deviation of the laboratory frame from the centre-of-mass frame is small (of relative order $\left|\vec{P}_{\Phi}\right| / E$ ) it may be neglected in the calculation of the RC. Within this approximation we define in this section the invariants

$$
s=S\left(\left|\vec{P}_{\Phi}\right|=0\right), \quad t_{1,2}=T_{1,2}\left(\left|\vec{P}_{\Phi}\right|=0\right)
$$

\subsection{Virtual and soft corrections}

The RC due to virtual photon emission can be computed employing the results of Ref. [18] (see also Ref. [19]) where the one-loop corrected Compton tensor with a heavy photon has been calculated for the scattering channel. In oder to obtain the corresponding results for the annihilation channel it is sufficient to make the substitutions

$$
p_{2} \rightarrow-p_{2}, u \rightarrow s, \quad s \rightarrow t_{2}, \quad t \rightarrow t_{1}
$$

in all formulae of Ref. [18].

In accordance with Ref. [18] the contribution to the differential cross-section for process (1) due to virtual and soft photon emission can be written as

$$
d \sigma^{V+S}=\frac{\alpha^{2}}{8 \pi^{3}} \sigma\left(q^{2}\right)\left[\rho L_{\mu \nu}^{\gamma}+T_{\mu \nu}\right] \widetilde{g}_{\mu \nu} \Theta\left(\frac{s-q^{2}}{4 E}-\omega_{m}\right) \frac{d^{3} k}{s \omega}
$$

where the quantity $\rho$ absorbs all infrared singularities. It can be presented as a sum of two contributions

$$
\rho=\rho_{V}+\rho_{S}
$$

where $\rho_{V}$ arises due to one-loop virtual corrections and $\rho_{S}-$ due to soft photon contributions. For the quantity $\rho_{V}$ we can use an expression derived in [18]

$$
\rho_{V}=4 \ln \frac{\lambda}{m}\left(L_{s}-1\right)-L_{s}^{2}+3 L_{q}+\frac{4 \pi^{2}}{3}-\frac{9}{2}, \quad L_{s}=\ln \frac{s}{m^{2}}, L_{q}=\ln \frac{q^{2}}{m^{2}} .
$$

Concerning the quantity $\rho_{S}$, the results of Ref. [18] are not valid, since in our case the experimental requirements for the softness of an additional photon inside the PD depend on its polar angle, see Eq.(5). Note that the parameters $\Delta_{1}$ and $\Delta_{2}$ in (5) are the physical ones and they will appear explicitly into the final expression for the RC. If an additional soft photon is outside the PD we can use an arbitrary small parameter $\Delta$ to define its maximum energy fraction. This parameter is an auxiliary one, and it disappears in the final result for the $\mathrm{RC}$ due to the possibility of an additional untagged hard photon emission outside the PD (see below).

When evaluating the soft photon corrections we present the corresponding cross-section in the factorized form

$$
d \sigma^{S}=d \sigma^{B} \delta, \quad \delta=-\frac{\alpha}{4 \pi^{2}} \int \frac{d^{3} k_{1}}{\omega_{1}}\left(\frac{p_{1}}{p_{1} k_{1}}-\frac{p_{2}}{p_{2} k_{1}}\right)^{2}, \quad \omega_{1}=\sqrt{\left(\vec{k}_{1}^{2}+\lambda^{2}\right)}
$$


where $k_{1}$ is the $4-$ momentum of an additional soft photon. Such factorized form is valid if $\sigma\left(q^{2}\right)$ is a smooth function of $q^{2}$, see Refs. $[15,20]$. In the case under consideration, the width of the $\rho$-resonance is large enough, and approximation (25) is justified.

One can verify, whether restriction (4) $(\Omega-|\vec{K}|<\eta)$ affects this form. Obviously, Eq.(25) is valid in the case of a very soft additional radiated photon. It is, therefore, sufficient to examine its impact for the maximum allowed energy of an additional soft photon. According to Eq.(5) this maximum energy is $\Delta_{2} E$. The above-mentioned restriction can be presented as

$$
\left(\omega+\omega_{1}-\eta\right)^{2}<\omega^{2}+\omega_{1}^{2}+2 \omega \omega_{1} \bar{c},
$$

where $\bar{c}$ is the cosine of the angle between the vectors $\vec{k}$ and $\vec{k}_{1}$. Since for the collinear photons $\gamma(k)$ and $\gamma\left(k_{1}\right)$ the constraint (4) is always fulfilled, one can check its validity for the maximal angle or for $\bar{c}=-1$. Setting $\bar{c}=-1$ and $\omega_{1}=\Delta_{2} E$ in the previous equation we obtain

$$
(2 \omega-\eta)\left(2 \Delta_{2} E-\eta\right)<0
$$

Therefore, we have

$$
\eta>2 \Delta_{2} E
$$

From Eqs.(4) and (5) it follows that this restriction is satisfied. Thus, we can use representation (25) in all angular phase space for an additional soft photon.

Integration of Eq.(25) with constraints (5) and

$$
\theta_{1}<\theta_{m}, \theta_{1}>\pi-\theta_{m}, \quad \omega_{1}<\Delta E
$$

leads to the following result for the $\mathrm{RC}$ due to soft photon emission

$$
\begin{gathered}
\delta=\frac{\alpha}{2 \pi} \rho_{S}, \\
\rho_{S}=\left[4\left(1-L_{s}\right) \ln \frac{\lambda}{\Delta m}+L_{s}^{2}-\frac{2 \pi^{2}}{3}+4\left(\ln \frac{\Delta_{1}}{\Delta} \ln \frac{1+c_{m}}{1-c_{m}}+\ln \frac{\Delta_{2}}{\Delta_{1}} \ln \frac{1+c_{1}}{1-c_{1}}+\ln \frac{\Delta}{\Delta_{2}} \ln \frac{1+c_{2}}{1-c_{2}}\right)\right],
\end{gathered}
$$

where $c_{1}=\cos 20^{\circ}$ and $c_{2}=\cos 40^{\circ}$ for the KLOE photon detector. Therefore, for the factor $\rho$, which is the sum of $(24)$ and (27) we have

$$
\rho=4\left(L_{s}-1\right) \ln \Delta+3 L_{q}+\frac{2 \pi^{2}}{3}-\frac{9}{2}+4\left(\ln \frac{\Delta_{1}}{\Delta} \ln \frac{1+c_{m}}{1-c_{m}}+\ln \frac{\Delta_{2}}{\Delta_{1}} \ln \frac{1+c_{1}}{1-c_{1}}+\ln \frac{\Delta}{\Delta_{2}} \ln \frac{1+c_{2}}{1-c_{2}}\right) .
$$

Note that quantity $\rho$ coincides with the well-known expression in the limiting case $\Delta_{i}=\Delta, i=$ 1,2 (see e.g. $[14,15,18]$ ).

Tensor $T_{\mu \nu}$ on the right-hand side of Eq.(23) has the structure

$$
T_{\mu \nu}=T_{g} \tilde{g}_{\mu \nu}+T_{11} \tilde{p}_{1 \mu} \tilde{p}_{1 \nu}+T_{22} \tilde{p}_{2 \mu} \tilde{p}_{2 \nu}-T_{12} \tilde{p}_{1 \mu} \tilde{p}_{2 \nu}-T_{21} \tilde{p}_{2 \mu} \tilde{p}_{1 \nu} .
$$

Now after carrying out the contraction of tensors on the right-hand side of Eq.(27) we arrive at

$$
\begin{gathered}
\frac{d \sigma^{V+S}}{d q^{2}}=\frac{\alpha}{2 \pi^{2}} \sigma\left(q^{2}\right) \frac{\left(s-q^{2}\right) d \cos \theta d \varphi}{4 s^{2}} \Theta\left(\frac{s-q^{2}}{4 E}-\omega_{m}\right) \\
\times \frac{\alpha}{2 \pi}\left[\rho \frac{\left(s+t_{1}\right)^{2}+\left(s+t_{2}\right)^{2}}{t_{1} t_{2}}+T\right] \\
T=\frac{3}{2} T_{g}-\frac{1}{8 q^{2}}\left[T_{11}\left(s+t_{1}\right)^{2}+\right. \\
\left.T_{22}\left(s+t_{2}\right)^{2}+\left(T_{12}+T_{21}\right)\left(s\left(s+t_{1}+t_{2}\right)-t_{1} t_{2}\right)\right], \quad s=4 E^{2} .
\end{gathered}
$$


As has been already mentioned for the KLOE detector $\left|t_{1,2}\right|>>m^{2}$, and therefore one can neglect all terms proportional to $m^{2}$ in the expressions for $T_{g}$ and $T_{i k}$. Then we obtain

$$
\begin{gathered}
T_{g}=-\left[\frac{s q^{2}}{t_{2}^{2}}+\frac{2 s\left(s+t_{2}\right)+t_{2}^{2}}{t_{1} t_{2}}\right] G+s\left(\frac{q^{2}}{t_{1} t_{2}}-\frac{2}{t_{1}+t_{2}}\right)\left(L_{q}-L_{s}\right)+\frac{s+t_{1}}{t_{2}}\left(\frac{3 s}{s+t_{2}}-1\right)\left(L_{q}-L_{1}\right)+(31) \\
\frac{s^{2}-t_{2}^{2}}{2 t_{1} t_{2}}+\left(t_{1} \leftrightarrow t_{2}\right), \\
T_{11}=\frac{2}{t_{1} t_{2}}\left\{-q^{2}\left(1+\frac{s^{2}}{t_{2}^{2}}\right) G-q^{2}\left(2+\frac{\left(s+t_{2}\right)^{2}}{t_{1}^{2}}\right) \widetilde{G}+2 q^{2}\left[\frac{\left(s+t_{2}\right)^{2}}{t_{1} t_{2}}+\frac{2 s}{t_{1}+t_{2}}\right]\left(L_{q}-L_{s}\right)+\right. \\
\frac{4}{t_{1}+t_{2}}\left[s^{2}-\left(s+t_{2}\right) t_{1}\right]\left[\frac{q^{2}}{t_{1}+t_{2}}\left(L_{q}-L_{s}\right)-1\right]+\frac{q^{2}\left(s+t_{2}\right)^{2}}{t_{1}\left(s+t_{1}\right)^{2}}\left(2 s+3 t_{1}\right)\left(L_{q}-L_{2}\right)+ \\
T_{12}+T_{21}=\frac{q^{2}}{t_{1} t_{2}}\left\{\frac{q^{2}}{t_{2}^{2}}\left(s+t_{1}\right)\left(s-t_{2}\right) G+\frac{q^{2}}{t_{1}^{2}}\left(s q^{2}-t_{1} t_{2}\right) \widetilde{G}-2 q^{2}\left(\frac{s q^{2}}{t_{1} t_{2}}+\frac{\left.\left.\left.2 s-t_{2}+t_{2}\right)-4 s-2 q^{2}+t_{1}-\frac{\left(s+t_{1}\right.}{s+t_{1}}\right\}\right)\left(L_{q}-L_{s}\right)-}{(33)}\right.\right. \\
\frac{4\left[s^{2}-\left(s+t_{1}\right) t_{2}\right.}{t_{1}+t_{2}}\left[\frac{q^{2}}{t_{1}+t_{2}}\left(L_{q}-L_{s}\right)-1\right]+\frac{q^{2}}{\left(s+t_{1}\right)^{2}}\left(2 s+3 t_{1}\right)\left(t_{2}-\frac{q^{2} s}{t_{1}}\right)\left(L_{q}-L_{2}\right)- \\
\left.\frac{q^{2}\left(s+t_{1}\right)}{t_{2}\left(s+t_{2}\right)}\left(2 s-t_{2}\right)\left(L_{q}-L_{1}\right)+8 s+3 t_{1}-t_{2}+\frac{2 s t_{2}}{s+t_{1}}\right\}+\left(t_{1} \leftrightarrow t_{2}\right),
\end{gathered}
$$

where the following notation has been introduced

$$
\begin{gathered}
G=\left(L_{q}-L_{s}\right)\left(L_{q}+L_{s}-2 L_{1}\right)+2\left[f(1)+f\left(1-\frac{q^{2}}{s}\right)-f\left(1-\frac{t_{1}}{q^{2}}\right)\right], \widetilde{G}=G(1 \leftrightarrow 2), \\
L_{1}=\ln \frac{-t_{1}}{m^{2}}, \quad f(x)=\int_{0}^{x} \frac{d t}{t} \ln (1-t) .
\end{gathered}
$$

The Born-like contribution (which is proportional to $\rho$ ) on the right-hand side of Eq.(30) absorbs all infrared singularities via the quantities $\ln \Delta, \ln \Delta_{1}$ and $\ln \Delta_{2}$. Concerning the collinear ones, the Born-like term, being integrated over the angular acceptance of the KLOE electromagnetic calorimeter, generates a contribution proportional to $\ln \theta_{m}$ while the remaining $T$-term - to both $\ln \theta_{m}$ and $\left(\ln \theta_{m}\right)^{2}$. This can be easily seen from studying the asymptotic behaviour of the term given in the second line on the right-hand side of Eq.(30), for instance, at small values of $\left|t_{1}\right|$. Neglecting the $\left|\vec{P}_{\Phi}\right| / E$ effects we obtain in this limiting case

$$
\left|t_{1}\right|=2 \omega E(1-c) \simeq E^{2} \theta_{m}^{2} \ll s,\left|t_{2}\right|, \quad t_{2}=-4 E^{2} x, q^{2}=4 E^{2}(1-x), \quad c=\cos \theta .
$$

Then we have

$$
\frac{\alpha}{2 \pi}\left\{2 \rho \frac{1+(1-x)^{2}}{x^{2}(1-c)}+\frac{2}{x(1-c)}\left[\frac{1+(1-x)^{2}}{x}\left(\ln (1-x) \ln \frac{x^{2}(1-c)}{2(1-x)}-2 f(x)\right)+\frac{2-x^{2}}{2 x}\right]\right\} .
$$




\subsection{Radiation of an untagged hard photon outside the PD}

When calculating $\mathrm{RC}$ due to the radiation of an additional invisible hard photon, we have to distinguish between the large $\left(140^{\circ}>\theta_{1}>40^{\circ}\right)$ and small $\left(\theta_{m}>\theta_{1}\right.$ and $\left.\theta_{1}>\pi-\theta_{m}\right)$ angle radiation. For large angles we take into account only the contribution proportional to $\ln \Delta$ and write it in the form

$$
\frac{d \sigma^{L}}{d q^{2}}=\frac{d \sigma^{B}}{d q^{2}} \frac{\alpha}{2 \pi} 4 \ln \frac{1}{\Delta} \ln \frac{1+c_{2}}{1-c_{2}}
$$

where the Born cross-section is defined by Eq.(12) with $\left|\vec{P}_{\Phi}\right|=0$.

To simplify the calculation of the small-angle contribution we use the quasireal electron approximation [21]. Of course all the experimental constraints for the event selection should be taken into account.

We begin with the general expression for the cross section describing the radiation of an untagged hard photon inside the small-angle blind zone

$$
\frac{d \sigma^{H}}{d q^{2}}=2 \frac{d \sigma_{s h}^{B}}{d q^{2}} \frac{\alpha E^{2}}{4 \pi^{2}}\left[\frac{1+(1-z)^{2}}{\left(k_{2} p_{1}\right)}-\frac{m^{2} z(1-z)}{\left(k_{2} p_{1}\right)^{2}}\right] d z d \cos \theta_{2} d \varphi_{2} \Theta_{\eta}
$$

where $z$ is the energy fraction of an untagged hard photon $z=\omega_{2} / E$,

$$
\begin{gathered}
\frac{d \sigma_{s h}^{B}}{d q^{2}}=\frac{d \sigma^{B}\left(p_{1}(1-z), k, p_{2}\right)}{d q^{2}}=\frac{\alpha}{\pi} \sigma\left(q^{2}\right) \frac{s(1-z)-q^{2}}{[2 E(2-z(1-c))]^{2}} \\
\times \frac{(1-z)^{2}\left(s+t_{1}\right)^{2}+\left((1-z) s+t_{2}\right)^{2}}{(1-z) t_{1} t_{2}} \frac{d \cos \theta}{(1-z) s} \Theta\left[\frac{s(1-z)-q^{2}}{2 E(2-z(1-c))}-\omega_{m}\right], \quad c=\cos \theta
\end{gathered}
$$

is the shifted Born cross-section (with the substitution $p_{1} \rightarrow(1-z) p_{1}$ ) and

$$
\Theta_{\eta}=\Theta\left(\left|\vec{k}+\vec{k}_{2}\right|-\left(\omega+\omega_{2}-\eta\right)\right)
$$

is the reduced form of the restriction

$$
\Omega-|\vec{K}|<\eta
$$

for the case of one untagged hard photon with the 4 -momentum $k_{2}=\left(\omega_{2}, \vec{k}_{2}\right)$. Factor 2 on the right-hand side of Eq.(38) appears because Eq.(37) describes collinear radiation of an additional hard photon along both electron and positron directions.

The $\Theta$-function on the right-hand side of Eq.(38) defines the maximum possible energy fraction $z_{\max }$ of an additional hard untagged photon emitted into the small-angle blind zone. It depends on the pion invariant mass $q^{2}$ and the polar angle $\theta$ of the hard photon hitting PD. Therefore it can be rewritten in the following form

$$
\Theta\left[\frac{s(1-z)-q^{2}}{2 E(2-z(1-c))}-\omega_{m}\right]=\Theta\left(z_{\max }-z\right), \quad z_{\max }=\frac{s-q^{2}-4 E \omega_{m}}{s-2 E \omega_{m}(1-c)} .
$$

The $\Theta_{\eta}$-function on the right-hand side of Eq.(37) leads to the nontrivial correlations between the limits for variables $z, \theta_{2}$ and $\varphi_{2}$. (Here we use the coordinate frame where $Z$-axis is the electron direction and define the $\left(\overrightarrow{p_{1}}, \vec{k}\right)$ plane as $\left.X Z\right)$. These limits can be understood in following way. First one needs to analyze the quantity

$$
B=\frac{z\left(1-c_{2}\right) E-\eta}{z s_{2} E}
$$


where $s_{2}=\sin \theta \sin \theta_{2}, c_{2}=\cos \theta \cos \theta_{2}$. If

$$
B>1
$$

then all azimuthal angles for an untagged hard photon are allowed, and we face two options for its polar angle and the energy fraction

$$
\begin{gathered}
I_{a}-\text { region : } 0<\theta_{2}<\theta_{m}, \Delta<z<\left[z_{\max }, \frac{\eta}{E\left(1-c_{+}\right)}\right] \\
I_{b}-\text { region }: \quad 0<\theta_{2}<\arccos \left(1-\frac{\eta}{z E}\right)-\theta, \frac{\eta}{E\left(1-c_{+}\right)}<z<\left[z_{\max }, \frac{\eta}{E(1-c)}\right] .
\end{gathered}
$$

where $c_{+}=\cos \left(\theta+\theta_{m}\right)$ and $[a, b]$ is $\min (a, b)$.

If the value of the quantity $B$ corresponds to

$$
1>B>-1
$$

then not all azimuthal angles for an untagged photon are allowed. In this case we obtain the following constraint

$$
0<\varphi_{2}<\arccos B, 2 \pi>\varphi_{2}>2 \pi-\arccos B
$$

The region defined by Eq.(42) is symmetric relative to the plane $(Z, X)$, which contains the momentum of the photon hitting the PD. For this case there are also two possibilities for the limits for $\theta_{2}$ and $z$

$$
\begin{aligned}
& I I_{a}-\text { region }: 0<\arccos \left(1-\frac{\eta}{z E}\right)-\theta<\theta_{2}<\theta_{m}, \frac{\eta}{E\left(1-c_{+}\right)}<z<\left[z_{\text {max }}, \frac{\eta}{E(1-c)}\right], \\
& I I_{b}-\text { region }=0<\theta-\arccos \left(1-\frac{\eta}{z E}\right)<\theta_{2}<\theta_{m}, \frac{\eta}{E(1-c)}<z<\left[z_{\text {max }}, \frac{\eta}{E\left(1-c_{-}\right)}\right],
\end{aligned}
$$

where $c_{-}=\cos \left(\theta-\theta_{m}\right)$.

Considering the integration limits defined by the relations (39)-(44) one can see that there is only one region $I_{a}$ in which the untagged photon energy fraction can reach its minimal value $\Delta E$. Because in this region all angles for the untagged photon are allowed we can perform an angular integration on the right-hand side of Eq.(37). The result reads

$$
\begin{gathered}
\frac{d \sigma^{H}\left(I_{a}\right)}{d q^{2}}=\frac{d \sigma^{B}}{d q^{2}} \frac{\alpha}{2 \pi}\left[-(4 \ln \Delta+3) L_{m}+4 \ln \Delta\right]+\frac{d \sigma_{1}^{H}}{d q^{2}} \\
\frac{d \sigma_{1}^{H}}{d q^{2}}=\int_{0}^{\left[z_{\max }, \frac{\eta}{E\left(1-c_{+}\right)}\right]} 2 \frac{d \sigma_{s h}^{B}}{d q^{2}} \frac{\alpha}{2 \pi}\left[P_{1}\left(1-z, L_{m}\right)-2 A(1-z)\right] d z, L_{m}=\ln \frac{E^{2} \theta_{m}^{2}}{m^{2}},
\end{gathered}
$$

where

$$
\begin{gathered}
P_{1}(x, L)=\lim \Delta \rightarrow 0\left[\frac{1+x^{2}}{1-x} \theta(1-x-\Delta)+\left(\frac{3}{2}+2 \ln \Delta\right) \delta(1-x)\right] L, \\
A(x)=\lim \Delta \rightarrow 0 \frac{x}{1-x} \theta(1-x-\Delta)+\ln \Delta \delta(1-x) .
\end{gathered}
$$

In Eq.(45) we separate the dependence of the contribution caused by the untagged hard photon emission on an auxiliary parameter $\Delta$. One can check explicitly that this term together with (36) cancels the $\Delta$-dependence of the the soft and virtual contribution(see Eqs.(27) and (30)). 
We can also perform the analytical angular integration for the contribution of the region $I_{b}$ on the right-hand side of Eq.(37)

$$
\begin{gathered}
\frac{d \sigma^{H}\left(I_{b}\right)}{d q^{2}}=\int_{\frac{\eta}{E\left(1-c_{+}\right)}}^{\left[z_{\max }, \frac{\eta}{E(1-c)}\right]} 2 \frac{d \sigma_{s h}^{B}}{d q^{2}} \frac{\alpha}{2 \pi}\left[\frac{1+(1-z)^{2}}{z} \ln (1+\gamma)-\frac{2(1-z) \gamma}{z(1+\gamma)}\right] d z \\
\gamma=\frac{E^{2}}{m^{2}}\left[\arccos \left(1-\frac{\eta}{z E}\right)-\theta\right]^{2} .
\end{gathered}
$$

Concerning the contribution of region $I I$ in Eq.(37), we can perform the corresponding analytical integration over the azimuthal angle only. For the remaining variables $\left(z, \theta_{2}\right)$ we will show the limits of integration

$$
\begin{aligned}
\frac{d \sigma^{H}(I I)}{d q^{2}}= & \left\{\int_{\frac{\eta}{E\left(1-c_{+}\right)}}^{\left[z_{\max }, \frac{\eta}{E(1-c)}\right]} d z \int_{\arccos \left(1-\frac{\eta}{z E}\right)-\theta}^{\theta_{m}} d \theta_{2}+\int_{\frac{\eta}{E(1-c)}}^{\left[z_{\max }, \frac{\eta}{E\left(1-c_{-}\right)}\right]} d z \int_{\theta-\arccos \left(1-\frac{\eta}{z E}\right)}^{\theta_{m}} d \theta_{2}\right\} \\
& \times \frac{2}{\pi} \arccos (B) \frac{d \sigma_{s h}^{B}}{d q^{2}} \frac{\alpha}{2 \pi} E^{2} \sin \theta_{2}\left[\frac{1+(1-z)^{2}}{\left(k_{2} p_{1}\right)}-\frac{m^{2} z(1-z)}{\left(k_{2} p_{1}\right)^{2}}\right] .
\end{aligned}
$$

Thus, the contribution in the $\mathrm{RC}$ due to the additional untagged hard photon emission is given by the sum of Eqs. (36), (45), (46) and (47).

\section{$5 \quad$ Full first-order radiative correction}

The first-order radiative correction to the cross-section for process (1) as measured by the KLOE detector with the above realistic experimental restrictions can be written as

$$
\begin{gathered}
\frac{d \sigma^{R C}}{d q^{2}}=\frac{\alpha}{\pi} \sigma\left(q^{2}\right) \frac{s-q^{2}}{4 s^{2}} d \cos \theta \Theta\left(\frac{s-q^{2}}{4 E}-\omega_{m}\right) \frac{\alpha}{2 \pi}\left\{T+\frac{\left(s+t_{1}\right)^{2}+\left(s+t_{2}\right)^{2}}{t_{1} t_{2}}\right. \\
\left.\times\left[3 \ln \frac{q^{2}}{s}+\frac{2 \pi^{2}}{3}-\frac{9}{2}+\left(3+4 \ln \Delta_{1}\right) \ln \frac{4}{\theta_{m}^{2}}+4 \ln \frac{\Delta_{2}}{\Delta_{1}} \ln \frac{1+c_{1}}{1-c_{1}}-\ln \Delta_{2} \ln \frac{1+c_{2}}{1-c_{2}}\right]\right\}+ \\
\frac{d \sigma_{1}^{H}}{d q^{2}}+\frac{d \sigma^{H}\left(I_{b}\right)}{d q^{2}}+\frac{d \sigma^{H}(I I)}{d q^{2}}
\end{gathered}
$$

where we used the approximation $\cos \theta_{m}=1-\theta_{m}^{2} / 2$.

The total cross-section $\sigma\left(q^{2}\right)$ of the one-photon annihilation process $e^{+} e^{-} \rightarrow \pi^{+} \pi^{-}$which should be extracted from the KLOE experiment measurements, is factorized on the right side of Eq.(48). This follows from the expression for $d \sigma_{s h}^{B} / d q^{2}$, see Eq.(38), which enters into each term in the third line in Eq.(48). This demonstrates an evident advantage of the approach of the Ref. [6] as compared with the scanning of the hadron cross-section by the tagged photon energy measurements. In the latter approach the RC caused by the additional invisible hard photon radiation include with necessity some integrals over $\sigma\left(q^{2}\right)[15,16]$. These integrals arise because in this case the tagged photon energy does not define the pion invariant mass directly. 
As noted above, the auxiliary parameter $\Delta$ disappears from the master formula (48) for the radiative correction to the cross-section of process (1). But all physical parameters, which define the event selection (namely: the "softness" parameters $\Delta_{1}$ and $\Delta_{2}$, angular parameters $\theta_{m}, \theta_{1}$ and $\theta_{2}$ as well as the energy threshold $\omega_{m}$ and parameter $\eta$ ) enter this formula either explicitly or via the integration limits in the third line. The differential cross-section over the measured $\pi^{+} \pi^{-}$-invariant mass $q^{2}$ is given by the sum of the Born term (17) which depends on the parameter $\left|\vec{P}_{\Phi}\right|$, and (48)

$$
\frac{d \sigma}{d q^{2}}=\frac{d \sigma^{B}}{d q^{2}}+\frac{d \sigma^{R C}}{d q^{2}}
$$

\section{Conclusion}

A crucial requirement for success of the forthcoming precision studies of the hadronic cross-section $\sigma\left(e^{+} e^{-} \rightarrow\right.$ hadrons $)$ at DA $\Phi$ NE through the measurements of radiative events $[6,7]$ is the matching level of reliability of the theoretical expectation. This, in turn, requires a detailed knowledge of the radiative corrections corresponding to the realistic conditions of the KLOE detector.

In this paper we derive the analytical expressions for the distribution over the invariant mass of the charged pion pair corresponding to the constraints of the proposed experiment [6] with the KLOE detector. When DA $\Phi$ NE operates at the $\Phi$ peak just this two-pion final hadronic state provides the dominant contribution to the ISR events due to the radiative return to the $\rho$ resonance. Our approach can be quite straightforwardly extended to the description of ISR events in the general case of an arbitrary hadronic final state.

Our formulae take into account both the kinematical constraints related to the geometry of the photon detector and the event selection cuts imposed in order to reduce the FSR contamination. When deriving the Born results the Lorentz boost of the $\Phi$ in the laboratory frame was accounted for. First of all, such an accuracy is essential for the high-precision determination of the tagged photon energy. For the purposes of calculation of the $\mathrm{RC}$ with the one per cent accuracy the $\left|\vec{P}_{\Phi}\right| / 2 E$ effects may be neglected.

A prospective advantage of the experimental strategy proposed in [6] is the direct precise determination of the two-pion invariant mass which, in turn allows to avoid the deconvolution procedure. One may even think about taking full advantage of the high precision of the measurements of the two charged pions with the drift chamber[6] by making the photon tagging redundant I. An obvious attractiveness of such an inclusive strategy is that the "invisible" ISR photons are then emitted dominantly in the very forward cones along the beams and the corresponding cross-sections are large (due to $\ln E^{2} / m^{2}$ enhancement). The overall event geometry becomes rather simple and the corresponding RC are governed by quasireal kinematics, see [21]. Then the constraints imposed by the performance of KLOE calorimeters become unimportant.

The corresponding results for the Born cross-section were presented in Ref. [14]. The derivation of the $\mathrm{RC}$ requires some modifications (as compared to the results given in Ref. [14]) due to the contribution from additional hard photon radiation, since, in this case, the invariant mass of the pions and not the energy of the tagged photon is measured.

However, the success of such an inclusive approach requires a special care regarding different background events. Thus, a carefully chosen event selection should be introduced in order to reduce as much as possible various contaminations such as FSR events, $\Phi \rightarrow \pi^{+} \pi^{-} \pi^{0} ; \pi^{+} \pi^{-} \gamma$ etc as well as double-photon mechanism of $\pi^{+} \pi^{-}$production. Further detailed examination of

\footnotetext{
${ }^{1}$ We are grateful to G. Venanzoni who has attracted our attention to such an option.
} 
the background caused by the strong decay modes and especially by the contribution of doublephoton $\pi^{+} \pi^{-}$production has to be performed. We plan to perform these studies elsewhere.

\section{Acknowledgements}

Authors thanks V.S. Fadin, G. Venanzoni and W. Kluge for fruitful discussion and critical remarks. N.P.M. thanks INFN for the hospitality. V.A.K. thanks the Leverhulme Trust for a Fellowship. This work was partly supported by the EU Framework TMR programme, contract FMRX-CT98-0194 (DG 12-MIHT) and CT-98-0169.

\section{References}

1. S. Aid et al., H1 Coll., Nucl.Phys. B 470 (1996) 3.

2. M.W. Krasny, W. Plazcek, H. Spiesberger, Z.Phys. C 53 (1992) 687.

3. D. Bardin, L. Kalinovskaya, T. Riemann, Z.Phys. C 76 (1997) 487;

H. Anlauf, A.B. Arbuzov. E.A. Kuraev, N.P. Merenkov, JETP Lett. 66 (1997) 391, ibid 67 (1998) 305; JHEP 9810 (1998) 013; Phys.Rev. D 59 (1999) 014003.

H. Anlauf, A.B. Arbuzov, E.A. Kuraev. In "Hamburg 1998/1999, Monte Carlo generators for HERA physics", p.539, hep-ph 9907248;

H. Anlauf, Eur. Phys. J. C 9 (1999) 69.

4. M. Benayoun, S.I. Eidelman, V.N. Ivanchenko, Z.K. Silagadze, hep-ph/9910523.

5. For recent publication see, for example, L3 Collab., M. Acciarri, et al., Preprint CERNEP/99-129, Sept. 1999.

6. G. Cataldi, A. Denig, W. Kluge, G. Venanzoni, KLOE MEMO 195, August 13, 1999.

7. S. Spagnolo, "La camera a drift di KLOE e prospettive e obiettivi di una nuova misura

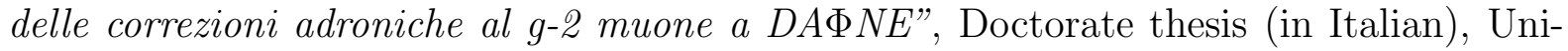
versity of Lecce, 1999, unpublished;

S. Spagnolo, Eur. Phys. J. C 6 (1999) 637.

8. S. Eidelman, F. Jegerlehner, Z. Phys. C 67 (1995) 585;

F. Jegerlehner, "Precision measurement of hadronic cross-sections at low energies", EURODA $\Phi$ NE Meeting, LNF, Frascati, Italy (1998);

F. Jegerlehner, "Hadronic effects in $(g-2)_{\mu}$ and $\alpha_{Q E D}\left(M_{Z}\right)$ : Status and perspectives, Preprint DESY 99-007, hep-ph/9901386;

F. Jegerlehner, "Sigma Hadronic and Precision Tests of the SM", LNF Spring School and EURODA $\Phi$ E Collaboration Meeting, Frascati, Italy, (1999).

9. N. Cabibbo, R. Gatto, Phys. Rev. 124 (1961) 1577.

10. CDM-2 Collaboration, R.R. Akhmetshin et al., Preprint Budker INP-99-10, hep-ex/9904027.

11. V.N. Baier, V.A. Khoze, Sov. Phys. JETP 21 (1965) 629, 1145. 
12. G. Pancheri, Nuovo Cim. A 60, 321 (1969);

M. Greco, G. Pancheri and Y.N. Srivastava, Nucl. Phys. B101, 234 (1975).

13. M.J. Greutz, M.B. Einhorn, Phys. Rev. D 9 (1970) 2537;

G. Bonneau, F. Martin, Nucl. Phys. B 27 (1971) 381;

M. Greco, Riv. Nuovo Cim. 11 (1988) 1.

14. A.B. Arbuzov, E.A. Kuraev, N.P. Merenkov, L. Trentadue, JHEP 12 (1998) 009.

15. M. Konchatnij, N.P. Merenkov, JETP Lett. 69 (1999) 811.

16. S. Binner, J.H. Kűhn and K. Melnikov, Phys. Lett. B 459 (1999) 279.

17. M. Caffo, H. Czyz and E. Remiddi, Nuovo Cim. 110 A (1997) 515; Phys. Lett. B 327 (1994) 369.

18. E.A. Kuraev, N.P. Merenkov, V.S. Fadin, Sov. J. Nucl. Phys. 45 (1987) 486.

19. A.B. Arbuzov et al., Nucl. Phys. B 485 (1997) 457.

20. E.A. Kuraev and V.S.Fadin, Sov. J. Nucl. Phys. 41 (1985) 466.

21. V.N. Baier, V.S. Fadin, V.A. Khoze, Nucl. Phys. 65 (1973) 381. 